\title{
Apoyo hacia la actividad física y rendimiento académico independientemente del estatus socioeducativo parental \\ Support towards physical activity and academic performance regardless of parental socio-educational status

\author{
Alberto Ruiz-Ariza, Manuel J. Dela Torre-Cruz, Sara Suárez-Manzano, Emilio J. Martínez-López
} \\ Universidad de Jaén (España)
}

\begin{abstract}
Resumen: El objetivo fue determinar si la influencia que los padres ejercen hacia la actividad física (AF) de los hijos está relacionada con el rendimiento académico (RA) en las asignaturas de Matemáticas y Lengua. Participaron 570 díadas padres-hijos/as. Se usó el cuestionario «The Parental Influence on Physical Activity ${ }^{\circledR}$ » y las calificaciones en Matemáticas y Lengua facilitadas por los Centros Educativos. Se emplearon análisis de regresión lineal y análisis ANCOVA controlando por edad, IMC, capacidad cardiovascular de los adolescentes, y estatus socioeducativo familiar. Los resultados mostraron que los chicos/as que reciben un mayor apoyo parental instrumental hacia la práctica de AF, y las chicas de padres más activos físicamente, tienen mejor RA en Lengua, independientemente del estatus socioeducativo familiar. El apoyo parental hacia la práctica de AF de los hijos podría ser un factor más a tener en cuenta para mejorar el RA de los adolescentes. Se sugiere a los padres la práctica habitual y conjunta de AF dentro del entorno familiar y procurar un apoyo instrumental sistemático hacia la AF de los hijos/as.
\end{abstract}

Palabras clave: Apoyo parental; rendimiento académico; estatus socioeducativo.

Abstract: The aim was to determine if parental influence on adolescent children's physical activity (PA) is related with academic performance (AP) in Mathematics and Spanish Language. A sample of 570 pairs of parents and children participated in this study. The Parental Influence on Physical Activity ${ }^{\circledR}$ questionnaire was used. Numerical marks in Mathematics and Spanish Language were requested from high schools. Linear regression and ANCOVA analysis were used for control of the variables (age, BMI, cardiovascular capacity of adolescents, and family socio-educational status). The results showed that adolescents with a higher parental instrumental support towards PA, and girls with higher physically active parents, have better AP in Spanish Language, regardless of family socio-educational status. Parental support towards children's PA may thus be another important factor in the promotion of AP in adolescents. It is suggested that parents practice PA within family context and provide systematic instrumental support towards their children's PA.

Keywords: parental support; academic achievement; socio-educational status.

\section{Introducción}

Durante la adolescencia, la influencia de los padres se considera un factor decisivo para el adecuado ajuste emocional y bienestar de los hijos (De la Torre-Cruz, Casanova, García, Carpio, \& Cerezo, 2011; Martínez-López, López-Leiva, Moral-García, \& De la Torre-Cruz, 2014; Oliva, Parra, \& Arranz, 2008). Estudios recientes han demostrado que el estilo parental educativo democrático - altos niveles de apoyo y de implicación hacia los hijos - está relacionado con comportamientos más saludables y un autoconcepto más positivo en los adolescentes (De la Torre-Cruz, Ruiz-Ariza, López-García, \& MartínezLópez, 2015; De la Torre-Cruz, Ruiz-Ariza, López-Serrano, \& MartínezLópez, 2018), una mayor competencia física atribuida (Berge, Wall, Larson, Loth, \& Neumark-Sztainer, 2013), y una mayor cantidad de actividad física (AF) diaria (Martínez-López et al., 2014).

Aunque esta última asociación ha sido poco estudiada, los efectos de la AF en jóvenes muestran una disminución significativa del estrés psicológico (Lavie, Milani, O’Keefe, \& Lavie, 2011), mayor felicidad, emociones positivas (Durán \& Costes, 2018), y motivación (Gutiérrez, Tomás, \& Calatayud, 2018), a la vez que un mayor rendimiento cognitivo (Esteban-Cornejo, Tejero-González, Sallis, \& Veiga 2015). Entre las posibles causas de este último efecto, se encuentra que la práctica de $\mathrm{AF}$ aumenta los niveles del factor neurotrófico derivado del cerebro y optimiza la plasticidad sináptica en los procesos neuroeléctricos de la corteza cerebral (Wrann et al., 2013). Ambos efectos favorecen la atención selectiva y la memoria, considerados factores clave para el rendimiento académico (RA) (Ruiz-Ariza, Grao-Cruces, Loureiro, \& Martínez-López, 2017b).

Pero el RA de los jóvenes está afectado además por otras variables biológicas como la edad, el índice de masa corporal (IMC), o la capacidad cardiovascular (Esteban-Cornejo et al., 2015). Factores parentales como la provisión de recursos, la aplicación de una correcta disciplina, el nivel de cohesión familiar o el nivel socioeducativo familiar (Caplan,

Fecha recepción: 19-10-17. Fecha de aceptación: 09-07-18

Alberto Ruiz Ariza

arariza@ujaen.es
Henderson, Henderson, \& Fleming, 2002), son también variables mediadoras en el RA de los jóvenes, siendo el nivel educativo de madre/ padres el que mejor representa el estatus socioeducativo de las familias (Castillo et al., 2011). Esta variable es válida, fiable y está sujeta a pocos cambios, siendo también la más empleada en estudios similares que asocian AF y cognición (Esteban-Cornejo et al., 2014; Ruiz-Ariza et al., 2017b). Los estudios previos revelan que el alumnado de familias con un nivel socioeducativo alto muestra actitudes más positivas hacia la escuela (Anderson, Sabatelli, \& Kosutic, 2007), un mejor comportamiento en clase (López, Olaizola, Ferrer, \& Ochoa, 2006) y, en definitiva, un mejor RA (Powers, Bowen, \& Rose, 2005). Por tanto, aislar el efecto socioeducativo es determinante para conocer la verdadera influencia de este tipo de apoyo parental. Nuestra búsqueda en Pubmed, Scopus, Web of Knowledge y SportDiscus, no ha encontrado ningún estudio que haya cuantificado la posible influencia sobre el RA de este apoyo en los hijos. Además, la mayoría de los estudios que investigan la influencia parental muestran resultados desde la percepción de los hijos (De la Torre-Cruz et al., 2011; De la Torre-Cruz et al., 2015), y en muy pocas ocasiones se presentan la opinión directa de los progenitores (Castillo et al., 2011).

Teniendo en cuenta toda lo anterior, el objetivo de este estudio residió en analizar la asociación entre el apoyo expresado por los padres/madres hacia la práctica de AF de los hijos con el RAen Matemáticas y Lengua, independientemente de la edad, IMC, capacidad cardiovascular y el estatus socioeducativo de las familias. También se examinó si un alto vs. bajo nivel de apoyo parental hacia la AF se relacionaba con mejores niveles de RA en estas asignaturas.

\section{Metodología}

\section{Participantes}

En el presente estudio transversal participaron un total de 570 diadas - padres e hijos/as adolescentes - de 12 Institutos de Educación Secundaria de Andalucía (España). Las características antropométricas, sociométricas y de RA de los hijos, así como las características de los padres se muestran en la tabla 1 y 2 respectivamente. 
Tabla 1

Tociońticas, capacidad cardiovascular, y rendimiento acánico de los adolescentes. Datos diferenciados por sexo.

\begin{tabular}{|c|c|c|c|c|c|c|c|}
\hline & \multicolumn{2}{|c|}{$\begin{array}{c}\text { Todos } \\
(\mathrm{n}=570)\end{array}$} & \multicolumn{2}{|c|}{$\begin{array}{c}\text { Chicos } \\
(\mathrm{n}=231)\end{array}$} & \multicolumn{2}{|c|}{$\begin{array}{l}\text { Chicas } \\
(\mathrm{n}=339)\end{array}$} & \multirow[t]{2}{*}{$p$} \\
\hline & Media & DT & Media & DT & Media & DT & \\
\hline Edad (años) & 14.12 & 1.43 & 14.03 & 1.43 & 14.18 & 1.42 & .198 \\
\hline Peso (kg) & 59.12 & 13.43 & 63.16 & 15.40 & 56.34 & 11.08 & $<.001$ \\
\hline Talla (m) & 1.64 & .09 & 1.68 & .09 & 1.61 & .07 & $<.001$ \\
\hline $\operatorname{IMC}\left(\mathrm{kg} / \mathrm{m}^{2}\right)$ & 21.91 & 4.03 & 22.19 & 4.34 & 21.71 & 3.81 & .174 \\
\hline $\begin{array}{l}\text { Capacidad cardiovascular } \\
\text { Course navette ( } \mathrm{n}^{\circ} \text { estadíos) } \\
\text { Rendimiento académico }(R A)\end{array}$ & 5.15 & 2.29 & 6.40 & 2.41 & 4.29 & 1.76 & $<.001$ \\
\hline $\begin{array}{l}\text { Rendimiento académico (RA) } \\
\text { RA en Matemáticas (1-10) }\end{array}$ & 6.35 & 2.08 & 6.19 & 2.20 & 6.46 & 1.99 & .146 \\
\hline RA en Lengua (1-10) & 6.48 & 1.93 & 6.08 & 2.05 & 6.76 & 1.79 & $<.001$ \\
\hline
\end{tabular}

Tabla 2

Características sociométricas de los padres e influencia parental (escala 1 = nunca; 4 = siempre) diferenciadas por sexo. Los datos se presentan como media, desviación típica (DT) y frecuencia (\%).

\begin{tabular}{|c|c|c|c|c|c|c|c|c|}
\hline & \multicolumn{2}{|c|}{$\begin{array}{c}\text { Todos } \\
(\mathrm{n}=570)\end{array}$} & \multicolumn{2}{|c|}{$\begin{array}{c}\text { Padres } \\
(\mathrm{n}=62) \\
\end{array}$} & \multicolumn{2}{|c|}{$\begin{array}{c}\text { Madres } \\
(\mathrm{n}=508)\end{array}$} & \multirow[t]{2}{*}{$p$} \\
\hline & & Media & DT & Media & DT & Media & DT & \\
\hline \multicolumn{9}{|c|}{ Características sociométricas } \\
\hline Edad (años) & & 44.16 & 4.81 & 46.70 & 4.17 & 43.85 & 4.79 & $<.001$ \\
\hline Peso $(\mathrm{kg})$ & & 69.38 & 12.85 & 83.82 & 15.3 & 67.37 & 11.10 & $<.001$ \\
\hline Talla (m) & & 1.63 & .07 & 1.74 & .07 & 1.62 & .06 & $<.001$ \\
\hline $\operatorname{IMC}\left(\mathrm{kg} / \mathrm{m}^{2}\right)$ & & 25.51 & 3.98 & 27.51 & 4.11 & 25.26 & 3.89 & $<.001$ \\
\hline \multirow{4}{*}{ Nivel de estudios (\%) } & Sin estudios & \multicolumn{2}{|c|}{2.8} & \multicolumn{2}{|c|}{1.6} & \multicolumn{2}{|c|}{3.0} & \\
\hline & Primaria & \multirow{2}{*}{\multicolumn{2}{|c|}{$\begin{array}{l}49.8 \\
24.3\end{array}$}} & \multirow{2}{*}{\multicolumn{2}{|c|}{$\begin{array}{l}41.9 \\
33.8\end{array}$}} & \multirow{2}{*}{\multicolumn{2}{|c|}{$\begin{array}{r}50.8 \\
23.3\end{array}$}} & \\
\hline & Secundaria & & & & & & & $<.001$ \\
\hline & Universidad & \multicolumn{2}{|c|}{15.3} & \multicolumn{2}{|c|}{17.7} & \multicolumn{2}{|c|}{15.0} & \\
\hline \multicolumn{9}{|c|}{ Influencia parental (1 - 4) } \\
\hline \multicolumn{2}{|c|}{ Apoyo instrumental } & 2.77 & .74 & 2.75 & .88 & 2.77 & .73 & .862 \\
\hline \multicolumn{2}{|l|}{ Actividad física presente } & 2.15 & .79 & 2.14 & .80 & 2.15 & .80 & .931 \\
\hline \multirow{2}{*}{\multicolumn{2}{|c|}{ Actividad física pasada }} & 2.17 & .93 & 2.22 & .85 & 2.16 & .94 & .628 \\
\hline & & 2.51 & .98 & 2.48 & .84 & 2.52 & .99 & .788 \\
\hline
\end{tabular}

\section{Instrumentos}

Variables predictoras

Influencia parental sobre la AF de los hijos/as

Para conocer la influencia de los padres sobre la AF de los hijos se empleóla adaptación del cuestionario «The Parental Influence on Physical Activity Scale ${ }^{\circledR}$ » compuesto por 14 ítems con escala tipo Likert de 1 (nunca) a 4 (siempre), que se agrupan en 4 factores (Jago, Fox, Page, Brockman, \& Thompson, 2009). Factor 1: Apoyo parental instrumental, referido al ánimo para que practique AF, dinero invertido, y acompañamiento a las instalaciones deportivas, entre otros. Factor 2: AF parental presente, referido a la práctica de $\mathrm{AF}$ actual de los padres. Factor 3: AF parental pasada. Y factor 4: Apoyo guiado, referido a las normas que los padres establecen en el hogar para facilitar la práctica de AF. Los índices de fiabilidad obtenidos mediante el estadístico alpha de Cronbach fueron: factor $1=.84$, factor $2=.86$, factor $3=.85$, $\mathrm{y}$ factor $4=.86$. El alpha total fue de .86 .

\section{Variable criterio}

Rendimiento Académico

Su registró se llevó a cabo utilizando la calificación en las asignaturas de Matemáticas y Lengua obtenidas en la evaluación trimestral previa a la cumplimentación del cuestionario (Ruiz-Ariza et al., 2016, 2017a).

\section{Variables de confusión controladas}

Edad e IMC de los hijos

Se consideró la edad informada por los Centros participantes en el momento de llevar a cabo la toma de datos. Para obtener las medidas de peso y talla se empleó una báscula digital ASIMED ${ }^{\circledR}$ modelo Elegant (Barcelona), y un tallímetro portátil SECA ${ }^{\circledR} 214$ (SECA Ltd., Hamburgo), respectivamente. Se calculó el IMC en $\mathrm{kg} / \mathrm{m}^{2}$ (MartínezLópez, Moreno-Cerceda, Suárez-Manzano, \& Ruiz-Ariza, 2018). Se usó el IMC por ser la variable de composición corporal más influyente sobre el RA (Esteban-Cornejo et al., 2015).

Capacidad cardiovascular

La capacidad cardiovascular se evaluó mediante el Test de 20 metros de ida y vuelta. Esta prueba está incluida en la batería ALPHAFitness ${ }^{\circledR}$ (Ruiz et al., 2011), y es considerada la variable de condición física más influyente a nivel académico (Ruiz-Ariza et al., 2017b).

Estatus socioeducativo de las familias

El estatus socioeducativo de las familias fue evaluado mediante el nivel de estudios de madres/padres, que fue autoinformado por los mismos (véase escala en la tabla 2). Del total de 570 participantes, contestaron 62 padres y 508 madres. Resultados de estudios previos han mostrado que la educación parental está altamente relacionada con el RA y es la medida que mejor representa el estatus socioeducativo familiar (Castillo et al., 2011; Esteban-Cornejo et al., 2014; Ruiz-Ariza et al., 2017b).

\section{Procedimiento de recogida y análisis de datos}

Se obtuvo el consentimiento informado de los responsables legales de los jóvenes. El nombre de padres e hijos/as fue codificado para asegurar el anonimato y confidencialidad. El estudio fue aprobado por la Comisión de Bioética de la Universidad de la Universidad de Jaén. Además, setuvieron en cuenta la Ley de Investigación Biomédica(2007), la Ley de Protección de Datos Personales (Ley Orgánica 15/1999), y los principios fundamentales de la Declaración de Helsinki (revisión 2013, Brasil).

Se comprobaron la normalidad y la homocedasticidad de las variables mediante el test de Kolmogorov y la prueba de Levene, respectivamente. Para la comparación de las variables en función del sexo se empleó la prueba t de Student para muestras independientes y el test $\chi^{2}$. Para estudiar las relaciones bivariadas entre variables se usó la correlación de Pearson. Los resultados de asociación de cada factor del cuestionario de influencia parental hacia la AF de los hijos/as con el RAse obtuvieron mediante análisis de regresión lineal. Para conocer si los adolescentes con baja ( $\leq$ Percentil 33) vs. alta ( $\geq$ Percentil 66) influencia parental sobre la AF tenían mejor o peor RA se realizaron análisis ANCOVA. Los análisis se realizaron por separado para chicos y chicas, ya que previamente se habían hallado interacciones significativas entre sexo al asociar la influencia parental con el RA en Lengua $(\mathrm{p}=.03)$. En todos los análisis se emplearon como covariables la edad, IMC, capacidad cardiovascular de los/as hijos/as, y estatus socioeducativo familiar. Se repitieron sin incluir en estatus socioeducativo para comprobar la importancia real de esta variable (datos no mostrados). Para todos los resultados se empleó un nivel de confianza del 95\% ( $<$.05). Los cálculos se realizaron con SPSS, v. 22.0 para Windows (SPSS Inc., Chicago).

\section{Resultados}

\section{Análisis de regresión lineal}

Los resultados del análisis de regresión lineal se presentan en la tabla 3 y 4. Tanto en chicos como chicas, ningún factor de influencia parental hacia la AF de los hijos se asoció con las calificaciones de Matemáticas (todos $\mathrm{p}>$.05). Solo el apoyo parental instrumental - factor 1- se correlacionó de forma bivariada con el rendimiento en Matemáticas $(r=.164$ y $r=.141$, para chicos y chicas respectivamente, ambas $\mathrm{p}<.05$ ). Respecto a la asignatura de Lengua, el apoyo parental instrumental - factor 1 - se asoció positivamente tanto en chicos $(\beta=$ $.582 \pm .224 ; p=.01)$ como en chicas $(\beta=.429 \pm .150 ; p=.005)$, independientemente de su edad, IMC, capacidad cardiovascular, y del nivel socioeducativo familiar. La AF diaria de los padres - factor 2 también se asoció positivamente con la calificación de Lengua, aunque solo en chicas $(\beta=.360 \pm .144 ; \mathrm{p}=.013)$. Y finalmente, la AF parental pasada - factor 3-y el apoyo guiado - factor 4- no se asociaron significativamente con las calificaciones de Lengua en chicos ni chicas ( $\mathrm{p}$ $>$.05). Por otra parte, encontramos que la capacidad cardiovascular se asociaba positivamente con las calificaciones en Matemáticas y Lengua en todos los factores, pero solo en las chicas (todos $\mathrm{p}<$.001). También se hallaron asociaciones entre el nivel socioeducativo familiar y las calificaciones en Matemáticas y Lengua de los chicos en los factores 2 , 3 y 4 (AF parental presente, pasada, y apoyo guiado, respectivamente, todos $\mathrm{p}<$.029). Y entre el nivel socioeducativo y el RA en Lengua de las chicas independientemente de la AF parental presente y apoyo guiado - factor 2 y 4 - (ambos $\mathrm{p}<.032$ ). Se repitieron los análisis sin el nivel socioeducativo de las familias, y los resultados de asociación entre los factores del apoyo parental y el RA, se incrementaron (datos no mostrados). No se hallaron asociaciones significativas respecto a la edad ni al IMC en los modelos propuestos ( $\mathrm{p}>.05)$. 
Tabla 3.

Análisis de regresión [valor de la Beta no estandarizada y error estándar (EE)] entre cada factor del cuestionario "The Parental Influence on Physical Activity", y las calificaciones en Matemáticas tras ajustar por edad, IMC, capacidad cardiovascular, y nivel socioeducativo familiar.

\begin{tabular}{|c|c|c|c|c|c|c|c|c|}
\hline & & & & Maten & náticas & & & \\
\hline & & hicos ( & $n=231$ & & & Chicas & $(n=33$ & \\
\hline & $B$ & $\mathrm{EE}$ & $\mathrm{r}$ & $p$ & $\beta$ & $\mathrm{EE}$ & $\mathrm{r}$ & $p$ \\
\hline$\overline{\text { Edad }}$ & -.090 & .136 & -.116 & .508 & -.061 & .089 & -.020 & .493 \\
\hline IMC & -.036 & .046 & -.026 & .436 & .034 & .036 & -.030 & .350 \\
\hline Capacidad cardiovascular & -.027 & .085 & .034 & .752 & .321 & .081 & $.225^{+*}$ & $<.001$ \\
\hline Nivel socioeducativo familiar & .258 & .138 & .189 & .064 & 184 & .113 & .182 & .107 \\
\hline Apoyo parental instrumental & .412 & .240 & $.164^{*}$ & .088 & .261 & .169 & $.141^{*}$ & .125 \\
\hline Edad & -.176 & .132 & -.116 & .186 & -.100 & .089 & -.020 & .265 \\
\hline IMC & -.031 & .045 & -.026 & .496 & .028 & 036 & -.030 & .448 \\
\hline Capacidad cardiovascular & .012 & .080 & .034 & .885 & .295 & 079 & $.225^{* *}$ & $<.001$ \\
\hline Nivel socioeducativo familiar & .320 & .136 & .189** & .020 & .165 & 1111 & .182 & .138 \\
\hline Actividad física presente & .163 & .205 & .088 & .429 & .224 & 161 & .102 & 164 \\
\hline Edad & -.186 & .131 & -.116 & .159 & -.074 & .090 & -.020 & .412 \\
\hline IMC & -.024 & .046 & -.026 & .605 & .040 & .036 & -.030 & .263 \\
\hline Capacidad cardiovascular & .010 & .080 & .034 & .898 & .344 & .080 & $.225^{*+}$ & $<.001$ \\
\hline Nivel socioeducativo familiar & .303 & .136 & $.189^{*-}$ & .027 & .167 & .112 & .182 & .139 \\
\hline Actividad física pasada & -.205 & .181 & -.049 & .259 & -.086 & .134 & -.048 & .520 \\
\hline Edad & -.204 & .131 & -.116 & .122 & -.069 & .090 & -.020 & .442 \\
\hline IMC & -.007 & .045 & -.026 & .879 & .041 & 036 & -.030 & .254 \\
\hline Capacidad cardiovascular & .043 & .080 & .034 & .587 & 346 & .079 & $.225^{*+}$ & $<.001$ \\
\hline Nivel socioeducativo familiar & .340 & 137 & $.189^{*+}$ & .014 & 180 & .112 & .182 & .111 \\
\hline Apoyo guiado & -.219 & .162 & -.105 & .177 & 156 & .133 & .026 & .242 \\
\hline
\end{tabular}

Tabla 4.

Análisis de regresión [valor de la Beta no estandarizada y error estándar (EE)] entre cada factor del cuestionario “The Parental Influence on Physical Activity” y la calificación en Lengua tras ajustar por edad, IMC, capacidad cardiovascular, y nivel socioeducativo familiar.

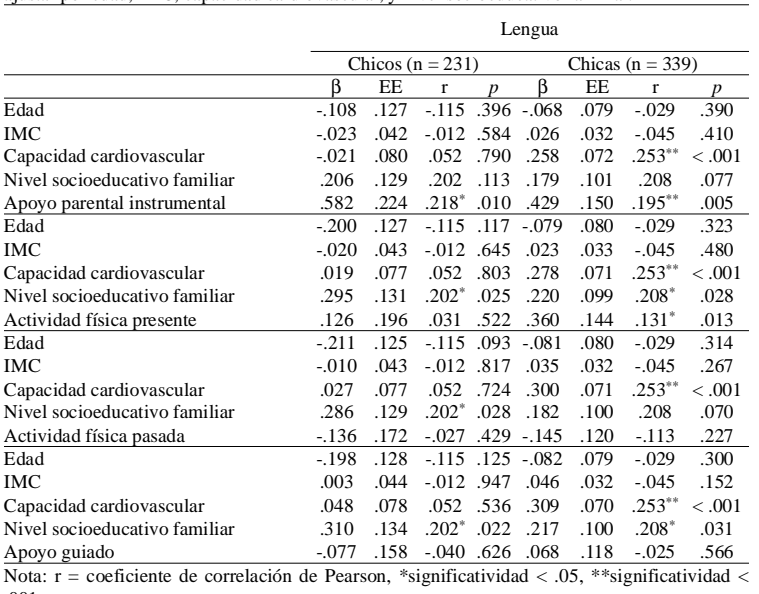
.001

Los resultados del análisis ANCOVA se muestran en la figura 1. Chicos y chicas con altos niveles de apoyo parental instrumental, alta AF actual y pasada de los padres, y un mayor apoyo guiado - factores

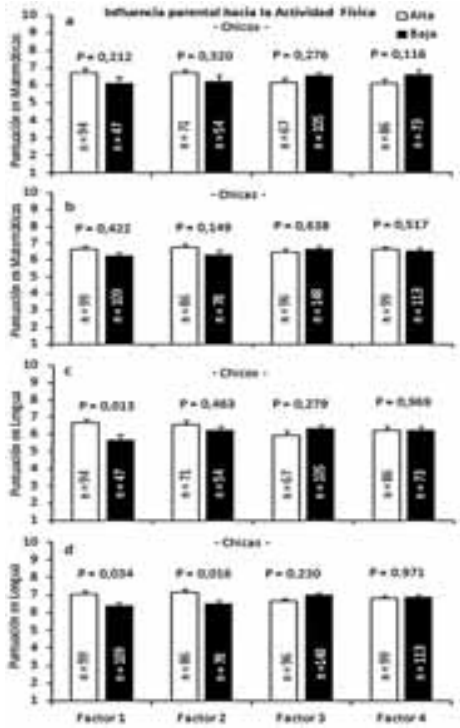

Figura 1

Análisis ANCOVA entre baja influencia parental hacia la AF ( $\leq$ Percentil 33) vs. alta influencia parental ( $\geq$ Percentil 66), y las calificaciones en las asignaturas de Matemáticas y Lengua. Se usó la edad, IMC, capacidad cardiovascular, y nivel socioeducativo familiar como covariables. Factor 1: Apoyo parental instrumental, factor 2: Actividad física parental presente, factor 3: Actividad física parental pasada, y factor 4: Apoyo parental guiado.
1, 2, 3, y 4, respectivamente - no mostraron mayores calificaciones en Matemáticas que aquellos con bajos niveles de influencia de los padres (todos $\mathrm{p}>.05$, fig. 1a,b). Respecto a la asignatura de Lengua, chicos y chicas con un alto apoyo parental instrumental hacia la AF - factor 1mostraron mejores calificaciones que quienes tenían un bajo apoyo $(\mathrm{p}=$ .013 y p $=.034$ respectivamente, fig. 1c, d). Asimismo, las chicas con padres que realizan una alta AF diaria - factor 2-mostraron calificaciones en Lengua significativamente superiores a las chicas de padres con baja AF ( $\mathrm{p}=.016$, fig. $1 \mathrm{~d})$. Una baja vs. alta AF parental pasada y apoyo guiado - factor 3 y 4 respectivamente - no influyó significativamente en el RA en Lengua ( $p>$.05).

\section{Discusión y conclusiones}

El objetivo de este estudio fue analizar la asociación del apoyo parental hacia laAF de los hijos adolescentes con el RAen Matemáticas y Lengua, independientemente del estatus socioeducativo familiar, entre otras variables mediadoras. Los resultados indican que el apoyo parental instrumental hacia la AF de los hijos está asociado de forma positiva con las calificaciones en Lengua, pero no en Matemáticas. Además, el RA en Lengua fue superior en las hijas de madres/padres que realizaban práctica habitual deAF. El RAen Matemáticas y Lengua no estaba asociado con la $\mathrm{AF}$ realizada por los padres en el pasado, ni con el apoyo guiado diario. Los anteriores hallazgos, se obtuvieron independientemente del estatus socioeducativo de las familias, que es una de las variables con mayor influye sobre el RA. Se repitieron los análisis sin tener en cuenta este factor, y todas las asociaciones aumentaron, demostrándose así la importancia de esta covariable y de los resultados obtenidos tras tenerla en cuenta.

No hemos encontrado trabajos que analicen la asociación entre influencia parental hacia la AF y RA, lo que impide que podamos hacer comparaciones directas. Sin embargo, el 73\% de los estudios revisados por Trost y Loprinzi (2011) informaron que el apoyo parental se asociaba positivamente con la práctica de AF en adolescentes. Otro estudio reciente mostró que la AF realizada en familia de forma conjunta, produce mejoras en el nivel de práctica de AF y en la condición física de los hijos (Cueto-Martín et al., 2018). Por su parte, otro trabajo mostró que más allá de variables personales como el sexo o el índice de masa corporal, la atribución de un elevado estilo transformacional parental se asoció con una mayor resistencia, fuerza, coordinación, apariencia y autoconcepto físico general percibidos por los hijos. Estos autores plantean la posibilidad de realizar intervenciones familiares en aras de incrementar los comportamientos característicos de este estilo de socialización parental (De la Torre-Cruz et al., 2018). Verloigne etal. (2014), hallaron que las creencias parentales sobre AF, y el apoyo instrumental - ánimo, dinero invertido, o acompañamiento a las instalaciones deportivas - mostrado a los hijos/as, se asociaban con la participación en AF de carácter organizado durante todos los días de la semana. En la misma línea, Morrissey, Wenthe, Letuchy, Levy y Janz (2012), concluyeron que el apoyo familiar - elogios a la participación y presencia física durante las actividades - era el mayor predictor de práctica de AF en adolescentes.

Nosotros hemos constatado que la influencia parental hacia la AF de los hijos sobre el RA es muy similar a los resultados obtenidos por Scudder et al. (2014). Estos hallaron que la práctica continuada deAF se relaciona positivamente con el RA en Lengua. También se asemejan a los resultados obtenidos por Sallis et al. (1999). Según este estudio longitudinal, los jóvenes que participaron en un programa de entrenamiento físico de dos años de duración obtenían mejores puntuaciones en Lengua y Lectura que quienes no lo hicieron. No obstante, esta relación directa entreAF y RA dista de la relación directa mostrada en el presente estudio, entre influencia parental hacia dicha práctica y RA.

La obtención de asociaciones significativas en la asignatura de Lengua pero no en Matemáticas añade controversia con respecto a trabajos previos. Algunos estudios han mostrado asociación entre AF y RA en Matemáticas (Moore et al., 2014), sin embargo, nuestros hallazgos difieren respecto a los anteriores y se asemejan a los obtenidos por 
Daley y Ryan (2000) y McNaughten y Gabbard (1993). Ambos estudios no hallaron asociación del tiempo de práctica diaria de AF moderada con las calificaciones de Matemáticas y con una prueba cronometrada de cálculo matemático, respectivamente.

Nuestros resultados también revelan que las chicas cuyos padres realizan más AF obtienen mejor RA en Lengua. Sin embargo, en chicos esta asociación no llegó a ser significativa. Wright, Wilson, Griffin y Evans (2010), destacaron que los chicos apreciaban niveles más elevados de apoyo instrumental que las chicas, además, solo las chicas indicaron recibir formas de apoyo no deseables, como sentirse forzadas a practicar deportes no deseados o a jugar con sus hermanos menores. Una posible explicación de las diferencias encontradas en función del sexo apuntaría a que las chicas con padres físicamente activos reciben una mayor transferencia de actitudes y hábitos de vida saludables (Berge et al., 2013), que podrían incrementar sustancialmente tanto la AF diaria como el RA. Además, la diferencia entre sexos también podría deberse al efecto dosis-respuesta (Martínez-Gómez et al., 2011). Es decir, durante la adolescencia las chicas muestran menos interés por la AF que los chicos y abandonan antes su práctica (Ruiz-Ariza et al., 2016). Por tanto, tal y como muestran nuestros datos y estudios recientes, un estímulo parental dirigido hacia el fomento de la práctica de $\mathrm{AF}$ podría tener un mayor efecto sobre el RA en las chicas que en los chicos (Esteban-Cornejo et al., 2015; Martínez-Gómez et al., 2011).

Adicionalmente, los análisis de regresión han revelado la importancia de algunas de las variables de confusión controladas, como el estatus socioeducativo familiar, una de las variables más influyentes en el RA (Esteban-Cornejo et al., 2014; Ruiz-Ariza et al., 2017b). Nuestros resultados muestran que un mayor estatus socioeducativo, se ha relacionado en chicos y chicas con mayores calificaciones de Matemáticas y Lengua, independientemente de la AF realizada por las madres/padres. Cuando repetimos los análisis sin incluir el estatus socioeducativo familiar, las asociaciones entre apoyo parental y RA aumentaron considerablemente, reflejándose el gran papel mediador de esta covariable. Por otro lado, una mayor capacidad aeróbica en las chicas se ha asociado con un mayor RA en Matemáticas y Lengua, independientemente de todos los factores de influencia parental hacia la AF. Por tanto, la capacidad cardiovascular y el nivel educativo de los progenitores son dos variables mediadoras muy importantes para el éxito académico.

Observamos que una de las limitaciones del estudio radica en el diseño transversal del mismo, lo queno permite establecer relaciones de causalidad. Además, las respuestas de los cuestionarios son opiniones de los padres que en algunos casos podrían haber sido contestados erróneamente, aunque esto podría haberse visto reducido por la codificación para asegurar el anonimato. Por el contrario, muy pocos estudios se han llevado a cabo sobre una muestra que implica simultáneamente a dos poblaciones relacionadas - padres e hijos-. También se han controlado covariables influyentes en el RA que han permitido independencia de los resultados.

Se concluye que, independientemente del estatus socioeducativo familiar, los chicos y chicas que reciben un mayor apoyo parental instrumental para practicar AF obtienen mejores calificaciones en Lengua pero no en Matemáticas. Asimismo, la práctica habitual de AF de los padres y madres no influye en las calificaciones de matemáticas de los hijos, pero síl lo hace positivamente en la calificación de Lengua de las hijas. Ni la AF llevada a cabo por los padres en el pasado, ni el apoyo guiado se ha asociado con un mayor RA de los hijos. Nuevos estudios longitudinales son necesarios para conocer las causas de las diferencias de RA entre asignaturas y entre sexos, y profundizar en los tipos de apoyo familiar que en mayor medida contribuyen al incremento de la práctica de la AF y de la condición física de los jóvenes, así como al aumento del RA. El apoyo parental para que los hijos/as practiquen AF podría ser un factor más a tener en cuenta en el fomento del RA durante la adolescencia. Se sugiere a madres/padres la práctica conjunta de AF con sus hijos/as, y reforzar el apoyo parental instrumental hacia la práctica de $\mathrm{AF}$.

\section{Agradecimientos}

El presente trabajo ha sido financiado parcialmente por el Proyecto «UJA2016/08/05-R3/8]» de la Universidad de Jaén, y por el Programa de Formación de Personal Docente Universitario del Ministerio de Educación, Cultura y Deporte(FPU-2014-01185). Los autores desean agradecer a los centros educativos, estudiantes y padres, su participación voluntaria en este estudio.

\section{Referencias}

Anderson, S.A., Sabatelli, R.M., \& Kosutic, I. (2007). Families, urban neighborhood youth centers, and peers as contexts for development. Family Relations, 56(4), 346-357. Doi:10.1111/ j.1741-3729.2007.00464.x

Berge, J. M., Wall, M., Larson, N., Loth, K. A., \& Neumark-Sztainer, D. (2013). Family functioning: Associations with weight status, eating behaviors, and physical activity in adolescents. Journal of Adolescent Health, 52(3), 351-357. doi:10.1016/ j.jadohealth.2012.07.006

Caplan, S. M., Henderson, C. E., Henderson, J., \& Fleming, D. L. (2002). Socioemotional factors contributing to adjustment among early-entrance college students. The Gifted Child Quarterly, 46(2), 124-134. doi.org/10.1177/001698620204600205

Castillo, R., Ruiz, J. R., Chillón, P., Jiménez-Pavón, D., EsperanzaDíaz, L., Moreno, L. A., \& Ortega, F. B. (2011). Associations between parental educational/occupational levels and cognitive performance in Spanish adolescents: theAVENAstudy. Psicothema, 23(3), 349-55.

Cueto-Martín, M. B., Cruz, J. C. de la, Morales-Ortiz, E., \& PérezDíaz, C. (2018). Effect of joint physical activity on the physical condition of parents and children. Journal of Human Sport and Exercise, 13(2), 415-429. Doi: 10.14198/jhse.2018.132.12

Daley,A. J., \& Ryan, J. (2000).Academic performance and participation in physical activity by secondary school adolescents. Perceptual \& Motor Skills, 91(2), 531-534. doi:10.2466/pms.91.6.531-534

De la Torre-Cruz, M. J., Casanova, P. F., García, M. C., Carpio, M. V., \& Cerezo, M. T. (2011). Estilos educativos paternos y estrés en estudiantes de educación secundaria obligatoria. Psicología Conductual, 19(3), 577-590.

De la Torre-Cruz, M. J., Ruiz-Ariza, A., López-García, M. D., \& Martínez-López, E. J. (2015) Efecto diferencial del estilo educativo materno y paterno sobre el autoconcepto físico del adolescente. Revista de Educación, 369, 59-84.

De la Torre-Cruz, M. J., Ruiz-Ariza, A., López-Serrano, S., \& MartínezLópez, E. J. (2018). La parentalidad transformacional se relaciona positivamente con el autoconcepto físico de jóvenes adolescentes. Retos, 34, 3-7.

Duran, C., \& Costes, A. (2018) Efecto de los juegos motores sobre la toma de conciencia emocional. Revista Internacional de Medicinay Ciencias de la Actividad Física y el Deporte, 18(70), 227-245. doi: 10.15366/rimcafd2018.70.003

Esteban-Cornejo, I., Tejero-González, C. M., Martinez-Gomez, D., del-Campo, J., González-Galo, A., Padilla-Moledo, C., . . \& \& Veiga, O. L. (2014). Independent and combined influence of the components of physical fitness on academic performance in youth. The Journal of Pediatrics, 165(2), 306-312.e2. doi:10.1016/ j.jpeds.2014.04.044

Esteban-Cornejo, I., Tejero-González, C. M., Sallis, J. F., \& Veiga, O. L. (2015). Physical activity and cognition in adolescents: Asystematic review. Journal of Science and Medicine in Sport, 18(5), 534-9. doi:10.1016/j.jsams.2014.07.007

Gutiérrez, M., Tomás, J. M., \& Calatayud, P. (2018). Determinantes de la práctica deportiva de los adolescentes en horario extraescolar. Revista Iberoamericana de Psicología del Ejercicio y el Deporte, 13, 91-100. 
Jago, R., Fox, K. R., Page, A. S., Brockman, R., \& Thompson, J. L. (2009). Development of scales to assess children's perceptions of friend and parental influences on physical activity. International Journal of Behavioral Nutrition and Physical Activity, 6, 67-76. doi:10.1201/b13134-7

Lavie, C. J., Milani, R. V, O’Keefe, J. H., \& Lavie, T. J. (2011). Impact of exercise training on psychological risk factors. Progress in Cardiovascular Diseases, 53(3), 464-470. doi:10.1016/ j.pcad.2011.03.007

López, E. E., Olaizola, J. H., Ferrer, B. M., \& Ochoa, G. M. (2006) Aggressive and nonaggressive rejected students: An analysis of their differences. Psychology in the Schools, 43, 387-400. Doi:10.1002/pits.20152

Martínez-López, E. J., Moreno-Cerceda, J., Suárez-Manzano, S., \& Ruiz-Ariza, A. (2018). Effect of and satisfaction with a program of physical activity controlled through heart rate monitors on body mass index in young students with overweight-obesity. Retos, 33, 179-184.

Martínez-López, E. J., López-Leiva, F., Moral-García, J. E., \& De la Torre-Cruz, M. J.(2014). Estilos educativos familiares e indicadores de actividad física en niños y adolescentes. Psicología Conductual, 22, 95-113.

Martínez-Gómez, D., Ruiz, J. R., Gómez-Martínez, S., Chillón, P., Rey-López, J., Díaz, L. E., \& Marcos, A. (2011). Active commuting to school and cognitive performance in adolescents: The AVENA Study. Archives of Pediatrics \& Adolescent Medicine, 165(4), 300305. doi:10.1001/archpediatrics.2010.244

McNaughten, D., \& Gabbard, C. (1993). Physical exertion and immediate mental performance of sixth-grade children. Perceptual \& Motor Skills, 77(3), 1155-1159. doi:10.2466/pms.1993.77.3f.1155

Moore, S. C., Patel, A. V., Matthews, C. E., Berrington de Gonzalez, A., Park, Y., Katki, H. A., ... \& Lee, I. M. (2012) Leisure time physical activity of moderate to vigorous intensity and mortality: a large pooled cohort analysis. PLOS Medicine, 9(11), e1001335. doi:10.1371/journal.pmed.1001335

Morrissey, J. L., Wenthe, P. J., Letuchy, E. M., Levy, S. M., \& Janz, K. F. (2012). Specific types of family support and adolescent nonschool physical activity levels. Pediatric and Exercise Science, 24(3), 333-346. doi:10.1249/01.mss.0000402543.07790.9c

Oliva,A., Parra, Á., \& Arranz, E. (2008). Estilos relacionales parentales y ajuste adolescente. Infancia y Aprendizaje, 31(1), 93-106. doi:10.1174/021037008783487093

Powers, J. D., Bowen, G. L., \& Rose, R. A. (2005). Using social environment assets to identify intervention strategies for promoting school success. Children \& Schools, 27, 177-185. doi:10.1093/cs/ 27.3.177
Ruiz-Ariza, A., de la Torre-Cruz, M. J., Suárez-Manzano, S., \& Martínez-López, E. J. (2017a). Active commuting to school influences on academic performance of Spanish adolescent girls. Retos, 32, 39-43.

Ruiz-Ariza, A., Grao-Cruces, A., de Loureiro, N., \& Martínez-López, E. J.(2017b). Influence of physical fitness on cognitive and academic performance in adolescents: Asystematic review from 2005-2015. International Review of Sport and Exercise Psychology, 10(1), 108133. doi:10.1080/1750984X.2016.1184699

Ruiz-Ariza, A., Ruiz, J., De la Torre-Cruz, M., Latorre-Román, P., \& Martínez-López, E. J. (2016). Influence of level of attraction to physical activity on academic performance of adolescents. Revista Latinoamericana de Psicologia, 48(1), 42-50. doi:10.1016/ j.rlp.2015.09.005

Ruiz, J. R., España, V., Castro, J., Artero, E. G., Ortega, F. B., Cuenca, M., . . \& \& Castillo, M. J. (2011). Batería ALPHA-Fitness: test de campo para la evaluación de la condición física relacionada con la salud en niños y adolescentes. Nutrición Hospitalaria, 26, 12101214.

Sallis, J., McKenzie, T., Kolody, B., Lewis, M., Marshall, S., \& Rosengard, P. (1999). Effects of health-related physical education on academic achievement: project SPARK. Research Quaterly Exercise \& Sport, 70(2), 127134. doi:10.1080/ 02701367.1999.10608030

Scudder, M. R., Federmeier, K. D., Raine, L. B., Direito, A., Boyd, J. K., \& Hillman, C. H. (2014). The association between aerobic fitness and language processing in children: implications for academic achievement. Brain and Cognition, 87, 140-52. doi:10.1016/ j.bandc.2014.03.016

Trost, S. G., \& Loprinzi, P. D. (2011). Parental influences on physical activity behavior in children and adolescents: A brief review. American Journal of Lifestyle Medicine, 5(2), 171-181. doi:10.1177/ 1559827610387236

Verloigne, M., Veitch, J., Carver,A., Salmon, J., Cardon, G., Bourdeaidhuij, I., \& Timperio,A. (2014). Exploring associations between parental and peer variables and physical activity among adolescents: A mediation analysis. BMCPublic Health, 14, 996. doi:10.1186/14712458-14-966

Wrann, C. D., White, J. P., Salogiannnis, J., Laznik-Bogoslavski, D., Wu, J., Ma, D., ... \& Spiegelman, B. M. (2013). Exercise induces hippocampal BDNF through a PGC-1á/FNDC5 pathway. Cell Metabolism, 18(5), 649-659. doi:10.1016/j.cmet.2013.09.008

Wright, M. S., Wilson, D. K., Griffin, S., \& Evans, A. (2010).Aqualitative study of parental modeling and social support for physical activity in underserved adolescents. Health Education Research, 25(2), 224-232. doi:10.1093/her/cyn
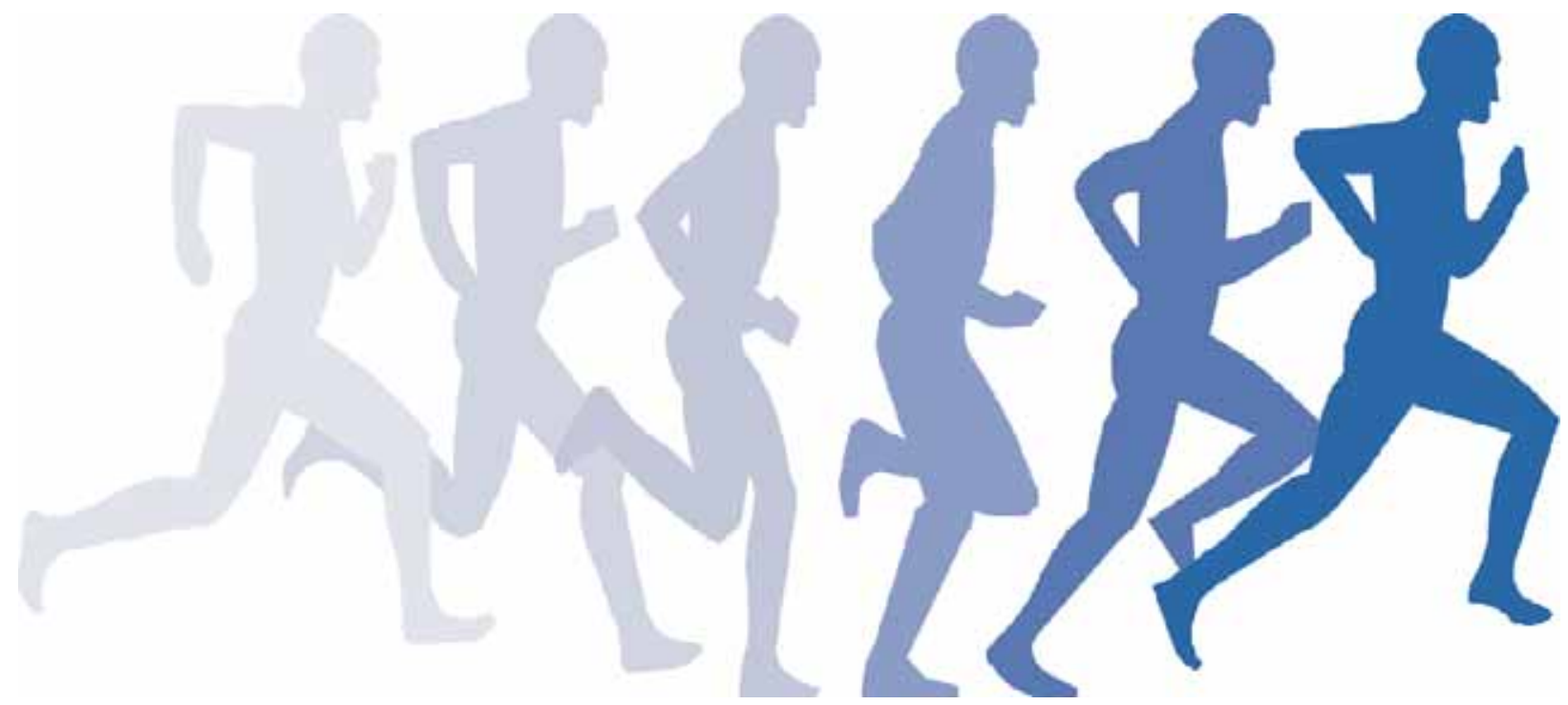